\title{
SYPHILITIC FOETUS AFTER PRENATAL PENICILLIN THERAPY*
}

\author{
BY \\ J. A. BURGESS \\ Royal Halifax Infirmary, Halifax
}

The prevention of congenital syphilis by the treatment of syphilitic pregnant women has been so successful that clinical manifestations of the disease in infants, following adequate prenatal penicillin therapy, are now seldom seen. The case described below has some unusual features.

\section{Case Report}

A 32-year-old woman, married for 14 years, attended an ante-natal clinic in her second pregnancy. Repeated serological tests for syphilis were positive and she was referred to a special treatment centre for examination and treatment. She gave a history of vaginal discharge and enlarged glands in the neck 9 years previously, but she had obtained no medical advice at that time and the condition had cleared up without treatment. Her first pregnancy occurred 2 years after her marriage and ended in the birth of a full-term healthy infant.

The sequence of events in her second pregnancy were as follows:

15th week: Attended ante-natal clinic. Specimen of blood taken.

16th week: First serological report received: Wassermann positive, Kahn positive, Price's precipitation reaction positive 1 in 32 .

17th week: Quickening felt.

19th week: Reattended ante-natal clinic. Specimen of blood taken.

20th week: Second serological report received-identical with first.

21st week: Foetal movements ceased. Examined at special treatment centre. No clinical signs of syphilis found.

22nd week: Crystalline sodium penicillin 1 mega unit daily for 15 days.

27th week: Miscarriage-macerated foetus.

A post-mortem examination of the foetal liver stained by Levaditi's method showed the presence of numerous spirochaetes which had the morphology of Treponema pallidum.

Examination of the husband showed that he was suffering from late latent syphilis.

\section{Commentary}

In this case, foetal movements ceased completely 7 days before penicillin therapy was started. The

\footnotetext{
${ }^{*}$ Received for publication June 25, 1958.
}

penicillin was freshly prepared before each injection and inquiries elicited no reason to doubt its potency. There can be little doubt that the foetus had died of syphilis in utero before penicillin was given.

It has been said, repeatedly, that congenital syphilis is a preventable disease, and that if all syphilitic pregnant women were treated adequately with penicillin there would be very few cases of infantile lues. This is undoubtedly true; but it may be that insufficient stress has been laid on the necessity of commencing treatment in pregnancy as soon as syphilis is provisionally diagnosed, and before the diagnosis has been confirmed.

At many ante-natal clinics the usual procedure, when a positive serological test for syphilis is reported, is to wait and take a second specimen of blood at the next visit of the patient to the clinic, which may be several weeks later. If the second specimen of blood is also positive, arrangements are made for further examination and treatment.

It would seem reasonable to suggest, to those undertaking ante-natal work, that, if the first specimen of blood gives an unequivocally positive result for syphilis, the expectant mother should be referred, immediately, to a special treatment centre where penicillin would be given without delay. Further investigation of the patient and her contacts would be undertaken, no doubt, while treatment was in progress. The course of action outlined above is justifiable in order to give the foetus, if infected with syphilis, the maximum chance of survival.

\section{Summary}

A case is described in which a pregnant woman received a course of penicillin injections, and was delivered 4 weeks later of a syphilitic foetus.

It is suggested that anti-syphilitic treatment should be given immediately to expectant mothers in whom the initial serological tests for syphilis are unequivocally positive.

I am indebted to Dr. N. Hamlin for information about the patient and to Dr. J. W. Weston for the pathological report. 